\title{
Correlations between Groups of Premotor Neurons Carry Information about Prehension
}

\author{
Eran Stark, ${ }^{1}$ Amir Globerson, ${ }^{2}$ Itay Asher, ${ }^{1,3}$ and Moshe Abeles ${ }^{1,3,4}$ \\ ${ }^{1}$ Department of Physiology, Hadassah Medical School, Hebrew University, Jerusalem 91120, Israel, ${ }^{2}$ Computer Science and Artificial Intelligence \\ Laboratory, Massachusetts Institute of Technology, Cambridge, Massachusetts 02139, ${ }^{3}$ Interdisciplinary Center for Neural Computation, Hebrew \\ University, Jerusalem 91904, Israel, and ${ }^{4}$ Gonda Brain Research Center, Bar-Ilan University, Ramat-Gan 52900, Israel
}

\begin{abstract}
How distinct parameters are bound together in brain activity is unknown. Combination coding by interneuronal interactions is one possibility, but, to coordinate parameters, interactions between neuronal pairs must carry information about them. To address this issue, we recorded neural activity from multiple sites in the premotor cortices of monkeys that memorized reach direction and grasp type followed by actual prehension. We found that correlations between individual spiking neurons are generally weak and carry little information about prehension. In contrast, correlations and synchronous interactions between small groups of neurons, quantified by multiunit activity (MUA), are an order of magnitude stronger. A substantial fraction of the information carried by pairwise interactions between MUAs is about combinations of reach and grasp. This contrasts with the information carried by individual neurons and individual MUAs, which is mainly about reach and/or grasp but much less about their combinations. The main contribution of pairwise interactions to the coding of reach-grasp combinations is when animals memorize prehension parameters, consistent with an internal composite representation. The informative interactions between neuronal groups may facilitate the coordination of reach and grasp into coherent prehension.
\end{abstract}

Key words: encoding; extracellular recordings; information theory; macaque monkey; multiunit activity; premotor cortex

\section{Introduction}

It is unknown how distinct concepts are combined into a composite mental representation. When several representations occur simultaneously, confusion may arise, generating a "property binding” problem (Treisman, 1996; Roskies, 1999). For example, when parameters such as color and shape of a composite object are encoded by different neurons, neural activity during the concurrent representation of a red circle and a blue square is ambiguous and might imply the presence of either red circle with blue square or red square with blue circle. Interactions between neurons may encode combinations of parameters: von der Malsburg (1981) suggested that correlations between neurons encoding distinct parameters occur during specific parameter combinations, when stimuli/behavioral requirements match the properties of the neurons (e.g., red-circle correlations occur when red and circle are parts of the same mental object). Although it was illustrated that synchrony may be stimulus specific (Eckhorn et al., 1988; Gray et al., 1989), how internal (nonsensory) composite mental representations actually form is still elusive.

If interneuronal correlations underlie composite internal rep-

\footnotetext{
Received July 21, 2008; revised Sept. 1, 2008; accepted Sept. 2, 2008.

This work was supported in part by Center of Excellence Grant 1564/04 administered by the Israel Science Foundation and the Deutsch-Israelische Projectkooperation. We thank Yoram Ben-Shaul, Rotem Drori, Gadi Goelman, Moshe Nakar, and Varda Sharkansky for help. We are grateful to Israel Nelken, Alit Stark, and Eilon Vaadia for critical comments.

Correspondence should be addressed to Eran Stark, Department of Physiology, Hadassah Medical School, Hebrew University, Jerusalem 91120, Israel. E-mail: eran.stark@ekmd.huji.ac.il.

D0I:10.1523/JNEUROSCI.3418-08.2008

Copyright $\odot$ 2008 Society for Neuroscience $\quad$ 0270-6474/08/2810618-13\$15.00/0
}

resentations, several conditions must be met. First, correlations should occur in the behaving animal, at behaviorally relevant timescales. Indeed, it is generally agreed that coarse temporal correlations [hundreds of milliseconds (Gawne and Richmond, 1993; Zohary et al., 1994; Lee et al., 1998; Reich et al., 2001)] and precise temporal synchrony [millisecond resolution (Toyama et al., 1981; Abeles, 1982a; Kwan et al., 1987; Kohn and Smith, 2005; Sakurai and Takahashi, 2006)] occur between cortical neurons. Second, to represent internal states, correlations should carry information even in the absence of stimuli or motor action. Although some studies suggested that the contribution of correlations to information processing is negligible (Shadlen and Newsome, 1998; Nirenberg et al., 2001), others demonstrated that correlations vary with sensory input (Gray et al., 1989; Laurent and Davidowitz, 1994; deCharms and Merzenich, 1996), motor output (Riehle et al., 1997; Maynard et al., 1999; Hatsopoulos et al., 2003), attention (Steinmetz et al., 2000), and past experience (Hoffman and McNaughton, 2002). However, it is unclear whether correlations vary independently of single-unit (SU) activity: recent studies have suggested that correlations between spiking neurons depend on SU properties including firing rates (de la Rocha et al., 2007; Tetzlaff et al., 2008). Thus, a critical issue in evaluating the information content of correlations is assessing the relationships between single-channel and pairwise codes. Third, to enable differentiation between several concurrent composite internal representations, correlations are expected to carry information about combinations of parameters. To date, this issue has hardly been addressed. 


\section{Prehension task}

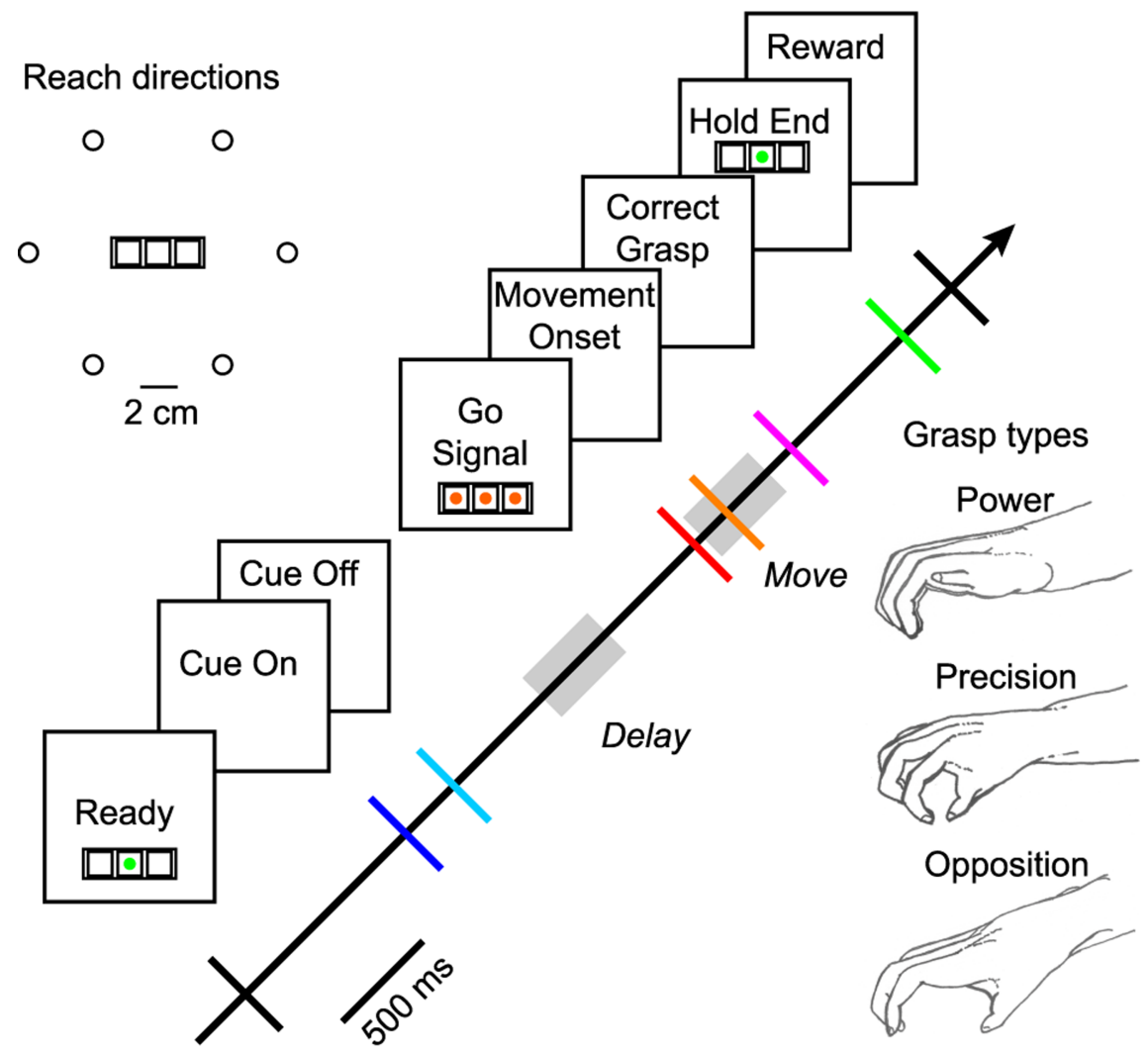

Figure 1. Prehension task. The timeline of the experimental paradigm is shown; gray bars show analysis epochs (see Materials and Methods). In each trial, an object was briefly presented in one of six locations arranged in a virtual circle around the central button of the touch pad.

Here, we studied correlations between pairs of SUs recorded from the macaque premotor cortex during memorization and execution of prehension. Because the spiking activity of local groups of premotor neurons [multiunit activity (MUA)] was shown recently to carry information about prehension (Stark and Abeles, 2007), we also estimated correlations between premotor MUAs. We found that correlations between MUAs carry information about prehension that does not exist in the individual MUAs and that their largest contribution is to the coding of reach-grasp combinations.

\section{Materials and Methods}

Animals and behavioral task. Two monkeys (female Macaca fascicularis, monkey D, $2.5 \mathrm{~kg}$ and monkey J, $3.2 \mathrm{~kg}$ ) were trained to perform unconstrained prehension movements with their right hand. The prehension task, recording procedures, and signal processing techniques have been described previously (Stark and Abeles, 2007; Stark et al., 2007) and are only briefly summarized below. All animal handling procedures were in accordance with the National Institutes of Health Guide for the Care and Use of Laboratory Animals (1996), complied with Israeli law, were approved by the Ethics Committee of the Hebrew University, and were supervised by a veterinarian.

During each session, six reach directions and two grasp types were used for a total of 12 reach-grasp combinations (Fig. 1). After the monkey pressed a button ("Ready") (Fig. 1), one object was shown at one location for 200-400 ms ("Cue on" to "Cue off"), indicating the upcoming reach direction and grasp type. After a delay of 1000-1500 ms (Cue Off to "Go Signal") during which the monkey was required to continue pressing the button and no visual stimulus was provided, a noninformative Go Signal prompted the monkey to reach, grasp, and hold the target object without visual feedback. During the delay and movement, the monkey could not see its hand or the object. Correct trials were reinforced by a juice reward. A typical session lasted $\sim 2 \mathrm{~h}$ during which monkeys completed 379 of these trials (median of 41 sessions; range of 164-585).

Recording procedures. Eye movements were monitored during all sessions (the Dr. Bouis Oculometer) (Stark et al., 2007). During some of the sessions, intramuscular EMGs were recorded by inserting pairs of wires into shoulder (acromion deltoid, latissimus dorsi, pectoralis major), elbow (biceps brachii, brachialis, triceps brachii), wrist (extensor carpii radialis longus, extensor carpii ulnaris, palmaris longus), and extrinsic digit (extensor digitorum communis, flexor digitorum profundus, flexor digitorum sublimis) muscles. The signals from these wires were amplified (30,000 times), bandpass filtered $(30-3000 \mathrm{~Hz})$, and sampled at $6.25 \mathrm{kHz}$. Up to eight muscles were monitored during each session; each muscle was recorded during 2-10 separate sessions, yielding a total of 61 recordings. Electrical crosstalk between concurrently recorded EMGs was assessed using the method of Kilner et al. (2002). First, the unrectified signals were smoothed (five-point moving average), down sampled to $1.25 \mathrm{kHz}$, and third-order differentiated. Then the correlation coefficient (CC) was computed between the third derivatives of every two concurrently recorded EMGs for time lags between -20 and $20 \mathrm{~ms}$. The analysis was applied to two 3 min segments of recording, from the beginning and end of each session, and crosstalk was detected if the maximum absolute CC over all time lags in either segment was above $0.1 ; 19$ muscle pairs were thus excluded from additional analyses. The root mean square (RMS) values of each EMG were then computed by raising signals to the second power, applying a $100 \mathrm{~Hz}$ low-pass filter, and taking the square root.

Premotor neural activity was recorded using up to 16 independently movable glass-coated tungsten microelectrodes (exposed tip size, 15-20 $\mu \mathrm{m}$; impedance, $0.2-2 \mathrm{M} \Omega$ at $1 \mathrm{kHz}$ ) arranged in two circular guide tubes (eight electrodes in each; minimal inter-electrode spacing within tube, $250 \mu \mathrm{m}$; Double MT; Alpha Omega Engineering). During each session, one tube was aimed toward arm-related (shoulder or elbow) regions of the dorsal premotor cortex (PMd) and another toward finger-related regions of the ventral premotor cortex (PMv). Forelimb relations were determined using sensory-motor mappings (SMMs), which consisted of two techniques: (1) threshold intracortical microstimulation (ICMS) $(0.2 \mathrm{~ms}$ biphasic pulses at $330 \mathrm{~Hz}$ for $90 \mathrm{~ms}$ at currents of 5-90 $\mu \mathrm{A})$, and (2) listening to the multiunit rustle of each electrode during proprioceptive, tactile, and visual input as well as during active movements (Stark et al., 2007). From the raw electrical signal recorded by each electrode (amplified 10,000 times, bandpass filtered at 1-10,000 Hz, and sampled at 25 $\mathrm{kHz}$ ), two measures of spiking activity were extracted: well isolated SUs and MUA. Spikes were detected in the raw signal using a modified second-derivative algorithm and sorted into SUs (Stark et al., 2007), each reflecting the activity of one neuron up to $\sim 30 \mu \mathrm{m}$ away from the recording electrode (Abeles, 1982a). MUA was estimated by the RMS (lowpass, $100 \mathrm{~Hz}$ ) of the bandpassed $(300-6000 \mathrm{~Hz})$ raw signal, reflecting the spiking activity of many neurons up to $\sim 100 \mu \mathrm{m}$ from the electrode (Grover and Buchwald, 1970; Stark and Abeles, 2007).

Neuronal database. Two epochs of $400 \mathrm{~ms}$ were used. The "Delay" epoch started $850 \mathrm{~ms}$ after Cue On and was thus in the middle of the delay period, and the "Move" epoch started $150 \mathrm{~ms}$ before "Movement Onset" and thus included both reaction time and movement time (Fig. 1, gray 
bars). For the analysis of precise temporal correlations, extended $1000 \mathrm{~ms}$ epochs were used, starting $450 \mathrm{~ms}$ after Cue On (Delay) or $150 \mathrm{~ms}$ before Movement Onset (Move). Qualitatively identical results were obtained for 200 and 800 $\mathrm{ms}$ epochs and at temporal offsets of $\pm 200 \mathrm{~ms}$ from those described above.

Individual channels (SUs, MUAs, and EMGs) considered for analysis were as follows: (1) well isolated, as determined by spike waveforms and by interspike interval histograms (applicable only to SUs); (2) recorded during at least 20 trials per reach-grasp combination exhibiting stationary activity [SUs: determined by visual inspection of mean firing rates and raster plots of individual trials; MUAs and EMGs: determined by fitting a straight line to the sequence of trial-by-trial activity and comparing its slope with those of lines fitted to randomly shuffled sequences (Paninski et al., 2004, their Appendix)]; (3) fired $>0.5$ spikes/s during both epochs (SUs) or the mean firing rate of all spikes on the recording electrode was $>1$ spikes/s (MUAs); and (4) recorded from a premotor site classified by ICMS and/or SMM as arm or finger related (applicable only to SUs and MUAs). Results were not sensitive to the specifics of these criteria, which conformed to those used in previous work (Stark and Abeles, 2007; Stark et al., 2007). In particular, identical results were obtained when MUAs were considered for analysis regardless of the spiking rate.

A total of 235 SUs formed 1289 jointly recorded pairs; each unit participated in 10 pairs (median; range, 1-24), and each pair was recorded during 341 common trials (median; range, 255-579). For the analysis of precise temporal correlations between SUs, the two units had to be recorded by different electrodes for at least 30 trials per reach-grasp combination, and the number of coincident spikes per bin had to be at least 0.1 (averaged over all time lag bins; see below). There were a total of 944 such pairs, comprising 162 units; each unit participated in 13 pairs (median), and each pair was recorded during 415 trials (median). A total of 142 MUAs formed 361 pairs, which were used for all analyses; each MUA participated in four pairs (median; range, $1-11$ ), and each pair was recorded during 319 trials (median; range, 251-565). Most SUs (156 of $235,66 \%$ ) and most MUAs (101 of 142, 71\%) were recorded from 101 common sites; there were no differences between channels recorded in common versus distinct sites. For the number of channels and channel pairs recorded within and across premotor regions (PMd and PMv), see Table 1.

Coarse temporal correlations. For each channel pair (SUs or MUAs), "signal correlations" and "noise correlations" (NCs) were estimated (Gawne and Richmond, 1993; Lee et al., 1998). For a given epoch, the signal correlation is the Pearson CC between the 12-element vectors of neural activity (spike count or MUA voltage), in which each element is the mean over all trials with the same reach-grasp combination. The NC is the CC between the vectors of neural activity, standardized to $Z$-scores (the mean subtracted and divided by the SD) for each reach-grasp combination separately. Denoting the standardized neural activity of channel $i$ during trial $t$ by $z_{i}(t)$, a single-trial correlation measure was obtained by the $Z$-score product, $z_{i j}(t)=z_{i}(t) \times z_{j}(t)$, and the $\mathrm{NC}$ by averaging over all available trials, $\left\langle z_{i j}(t)\right\rangle_{t}$. A combination-specific CC was obtained by averaging $z_{i j}$ over the subset of trials in the corresponding reach-grasp combination (see Fig. 3B).

Two control calculations were made on NCs to determine whether their values were attributable to slow temporal comodulations and/or correlations with parameters other than reach-grasp. First, to account for slow temporal comodulations, we computed NCs after standardizing the neural activity of each channel to $Z$-scores using a time-varying mean and SD. These were estimated by fitting, separately for each reach-grasp combination, a polynomial function to the trial-by-trial sequence of spike counts (or MUA voltage). The polynomial order is critical: a zeroorder polynomial yields a constant mean and $\mathrm{SD}$, ignoring potential temporal variations, whereas an order equal to the number of trials overcompensates for such variations. To prevent overfitting, we used twofold cross-validation (the folds being odd and even trials) and chose the polynomial order that minimized the generalization error. Second, to account for correlations with potentially confounding factors, including reaction time, movement time, gaze angle, and muscle activity, we computed partial correlations between two vectors of standardized neural activity, given the standardized values of the confounders. The resulting NCs were nearly unaltered by these manipulations, and thus the "raw" NCs are reported in Results.

Precise temporal correlations. To estimate precise temporal correlations between SU spike trains, we adapted the method of Abeles and Gat (2001) to pairwise correlations. Specifically, to keep the counting process Poissonian, individual spike trains were first diluted by deleting all spikes except the first one whenever an interspike interval was below $6 \mathrm{~ms}$; this procedure removed $3 \pm 0.3 \%$ of the spikes (mean \pm SEM over 162 SUs). Cross-correlation histograms (CCHs) were estimated at a $1 \mathrm{~ms}$ resolution by counting the number of times the two units fired spikes at exactly the same millisecond and at any time lag between -100 and $100 \mathrm{~ms}$ one relative to another. A predictor $\mathrm{CCH}$ was then computed by convolving the raw $\mathrm{CCH}$ with a finite kernel (partially hollowed Gaussian, SD of 5 $\mathrm{ms}$, with the central bin set to $40 \%$ of the original value), based on the null hypothesis that spike timing does not matter up to a given temporal resolution. The probability of obtaining an observed (or larger) count in the raw $\mathrm{CCH}$ was then estimated from the predictor $\mathrm{CCH}$ using the Poisson distribution with a continuity correction (Abeles and Gat, 2001). Finally, the difference $\mathrm{CCH}$, obtained by subtracting the predictor $\mathrm{CCH}$ from the raw $\mathrm{CCH}$ was divided by the recording duration to measure the excess coincidence rate (see Fig. $6 \mathrm{~A}$ ).

We verified the objectivity of this procedure in two ways. First, we generated artificial pairs of spike trains (independent Poisson processes, 5 spikes/s, 100 trials of $1 \mathrm{~s}$ ) and subjected them to exactly the same procedure as the recorded trains. Second, we used real spike trains and shuffled the trial order of one of the units, separately for each reachgrasp combination [conditionally independent (CI) shuffling; see below]. In both cases, the difference CCHs and the distributions of $p$ values were flat. In particular, the $p$ value distributions for the zero-lag bin were uniform.

In contrast to spike trains which are point processes, MUAs are continuous-time signals. To obtain an estimate of precise temporal correlation that is not influenced by slower comodulations of two MUAs, individual channels were first high-pass filtered $(10 \mathrm{~Hz}$, three-pole Butterworth). For each trial separately, the correlation between two simultaneously recorded MUAs was then estimated by computing the CC at time lags from - 100 to $100 \mathrm{~ms}$; for computational efficiency, lagged CCs were computed in the frequency domain using fast Fourier transform. The probability of obtaining an observed (or larger) trial-averaged CC was estimated by a $t$ test on the single-trial Fisher's $z$-transformed CCs; identical results were obtained using a permutation test.

Mutual information between neural activity and reach-grasp behavior. To quantify coding of motor behavior by neural activity, we estimated the mutual information (MI) between them. By motor behavior, we refer to memory or execution of reach and/or grasp, and, by neural activity, we refer to any aspect of neural activity, including SU spike counts or MUA 
A

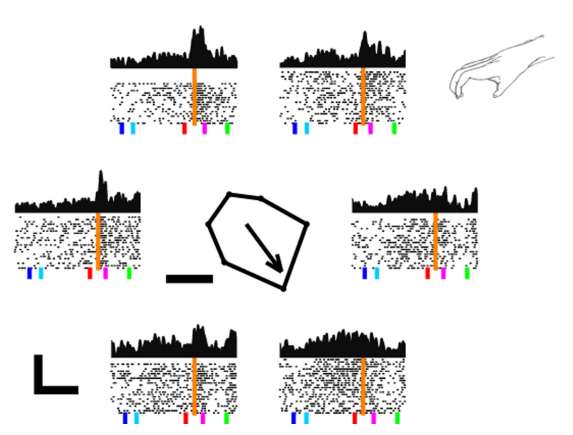

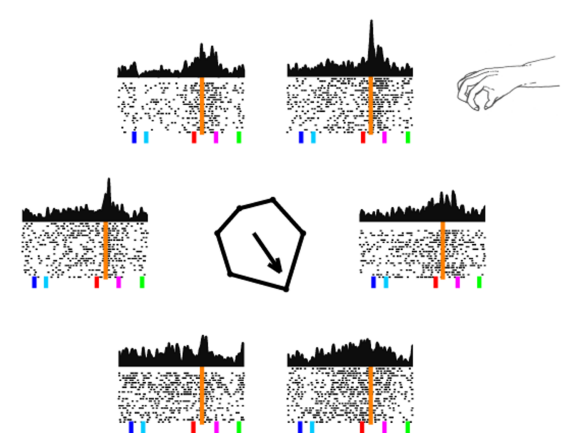

B

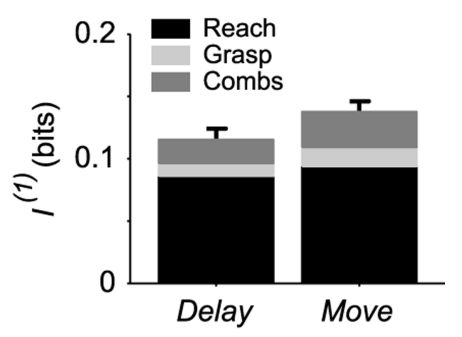

Figure 2. SU properties during prehension. A, Activity of a PMd unit during the prehension task. Left, Activity during finger opposition trials. Right, Activity during precision grip trials. Each small panel in a set of six shows rasters (bottom; aligned on Movement Onset, orange vertical lines) and perievent time histograms (top; smoothed with a Gaussian kernel, SD of $15 \mathrm{~ms}$ ) in the direction corresponding to the actual movements. Mean event times are shown at the bottom of each panel (blue, Cue On; light blue, Cue Off; red, Go Signal; pink, Correct Grasp; green, Hold End). Calibration: $1 \mathrm{~s}, 25$ spikes/s. Polar plots in centers show mean firing rates (scale bar, $10 \mathrm{spikes} / \mathrm{s}$ ) and arrows show preferred directions during the delay; reach directions were similar during memorization of the two grasp types. B, Bars show the total MI carried by the spike count code, averaged over all premotor SUs that carry Ml about prehension (Delay, 136 of 235 units; Move, 180 of 235 units); error bars indicate SEM. Segments in grayscale show the MI about reach, grasp, and their combination, averaged over all of the units. Most SU information was about reach direction.

voltage (denoted as $I^{(1)}$ ), coarse temporal correlations between two channels or pairwise synchrony $I^{\text {(corr) }}$, and the overall activity of two channels, including their individual activity and the interactions between them, $I^{(1,2)}$. The total MI between neural activity and reach-grasp behavior, $I_{\text {total }}$, can be decomposed into a sum of three terms:

$$
I_{\text {total }}=I_{\text {reach }}+I_{\text {grasp }}+I_{\text {comb }} \text {. }
$$

All terms are measured in bits and are non-negative. For the full approach and mathematical proof, see Appendix. Briefly, $I_{\text {total }}$ quantifies the information carried by the neural activity about prehension (12 behaviors), $I_{\text {reach }}$ measures the MI on reach (six directions), and $I_{\text {grasp }}$ measures the MI on grasp (two types). $I_{\text {comb }}$ measures the information on nonlinear combinations: the coding of reach and grasp that is beyond coding of reach and, simultaneously and independently, coding of grasp. For instance, an extreme example of such coding would be a neuron that fires only when movement is to the right in association with a power grip and only when movement is to the left in association with a precision grip. Nonlinear combination coding can be used to distinguish between simultaneous representations (or motor strategies) to act on two goals and is of key interest in the current work.

To measure the information in pairwise activity that is contributed specifically by pairwise interactions between two channels, we compared the information provided by the joint activity of the two channels, denoted as $I^{(1,2)}$, to the information assuming that the two channels are conditionally independent, denoted as $I^{(1,2) \mathrm{CI}}$. For methodological considerations and computational specifics, see Appendix. The difference between each of the $I^{(1,2)}$ and the corresponding $I^{(1,2) C I}$,

$$
\Delta I_{\text {total }}^{(1,2)}=I_{\text {total }}^{(1,2)}-I_{\text {total }}^{(1,2) \mathrm{CI}}
$$

(with equivalent expressions for $\Delta I^{(1,2)}$ reach $\Delta I^{(1,2)}$ grasp, and $\Delta I^{(1,2)}$ comb), quantifies the increase/decrease in information on each parameter that results from higher-order interactions between the two channels. $\Delta I^{(1,2)}$ can capture information that does not exist in linear correlations available to the $I^{\text {(corr) }}$ measure such as source identity (which neuron fired how many spikes) and higher-than-second-order interactions. In addition, the $\Delta I^{(1,2)}$ measure can be positive, for instance when pairwise interactions are modulated by behavior in a consistent manner, or negative, for instance when pairwise interactions are identical for all behaviors and the signal and noise correlations have the same sign. Similar measures based on the CI reference have been used in previous work: $\Delta I^{(1,2)}$ is identical to the sum of the $I_{\text {cor-ind }}$ and $I_{\text {cor-dep }}$ measures (Pola et al., 2003) (these two measures should not be confused with the $I^{\text {(corr) }}$ used here). $I_{\text {cor-ind }}$ can be positive or negative, whereas $I_{\text {cor-dep }}$ is nonnegative and identical to the $\Delta I$ measure of Nirenberg et al. (2001).

To quantify the portion of the overall pairwise information contributed by pairwise interactions, we computed the fractional $\Delta I^{(1,2)}$ :

$$
f \Delta I^{(1,2)} \text { total }=\Delta I_{\text {total }}^{(1,2)} I^{(1,2)} \text { total }
$$

with equivalent expressions for reach, grasp, and combinations. $f \Delta I^{(1,2)}$ is 0 when pairwise interactions do not contribute to the overall pairwise information, 1 when all information comes from pairwise interactions, and negative otherwise.

Statistical testing. For a given effect size, the power of any (resampling or conventional) statistical test depends on the $\alpha$ level. To increase the sensitivity of detecting effects, we report results based on a significance threshold of 5\%; all results were maintained when $\alpha=1 \%$ was used instead.

A standard bootstrap procedure was used to test whether measures whose distributions do not have predefined forms (e.g., Spearman's rank correlation, $f \Delta I^{(1,2)}$ ) differ from 0 . We resampled the population with replacement 1000 times, computed the median value of each resampled repetition, and measured the fraction of resampled medians that were nonpositive (a one-sided test).

Two permutation tests were used for external verification of the information analyses. The first test was a "mixing" test, and the second was a "CI shuffling" test. In the mixing test, we randomly shuffled the behavioral tags between trials (resampling without replacement) and repeated the entire procedure. This manipulation is expected to reduce the biascorrected information in any neural code to $\sim 0$ and yield chance distributions of chance information. We performed this test for all information analyses; chance level information was indeed obtained in all cases (data not shown). The CI shuffling test was applied only to analyses of pairwise neural codes. In this test, we randomly shuffled (resampling without replacement) the trial order of one channel (SU or MUA) relative to the other, separately for each reach-grasp combination. In contrast to mixing, this manipulation does not alter the MI in the individual channels but is expected to remove all information carried and/or contributed by pairwise interactions. The outcomes of CI shuffling are reported in the appropriate sections of Results.

\section{Results}

Single premotor units carry little information about reach-grasp combinations

A prehension trial began with a brief presentation of a target object in one of six directions relative to a central touch pad (Cue On) (Fig. 1). Two different objects requiring different grasp types were used during each session, resulting in 12 reach-grasp combinations. A memory period of at least $1 \mathrm{~s}$ followed, during which the monkey was required to keep its hand still on the touch pad and no stimulus was provided. Subsequently, a noninformative Go Signal prompted the monkey to reach and grasp the target. An example of SU activity during this task is shown in Figure 2A. During memorization (Delay epoch), the unit tended to fire more spikes at trials in the bottom right directions, regardless of grasp type. The total MI between the spike count code of this 
example unit and prehension type (one of 12 possible reach-grasp combinations) during Delay was 0.081 bits; $>90 \%$ of this information ( 0.079 bits) was about reach direction. Of 235 SUs analyzed in this way, $136(58 \%)$ were informative about prehension (chance level here and in all following tests is 5\%). The fractions of informative units in PMd and PMv were similar (Table 1, first row). During movement execution (Move epoch), the fraction of informative SUs was higher than during Delay $\left(77 \%\right.$; $\chi^{2}$ test, $\left.p \ll 0.001\right)$, but, in both premotor regions during both epochs, most of the MI carried by informative SUs was about reach direction or grasp type (Fig. 2B).

Individual forearm muscles had an even lower tendency to code reach-grasp combinations than SUs: whereas all 61 muscle recordings were informative during Move, $10.3 \pm 1.4 \%$ (mean \pm SEM) of the information was about reach-grasp combinations compared with $21 \pm 1.6 \%$ for SUs during the same epoch. Thus, only a small fraction of the information carried by forearm muscles and premotor SUs was about reach-grasp combinations.

Coarse temporal correlations between

SUs vary with reach and grasp in a manner consistent with firing rate variations

Over 1289 simultaneously recorded SU pairs, NCs (Gawne and Richmond, 1993) (see Materials and Methods) were biased to positive values (medians, 0.014 and 0.011 ; bootstrap tests comparing medians with $0, p<0.001)$. This bias did not result from slow temporal drifts or from correlations with parameters such as reaction time, movement time, eye movements, or muscle activity (see Materials and Methods). Although some SU pairs had NCs as high as 0.49 , NCs were typically small, and only 66 of $1289(5 \%)$ of the NCs were above 0.15. Consistent with studies on other brain regions (Lee et al., 1998), NCs between premotor neurons were higher for pairs with similar mean activity patterns (signal correlations; stronger during Delay than during Move; permutation test, $p<0.01$ ) as well as for nearby SUs (Fig. 3A, left two panels). There was no reliable relationship between NCs and similarity of the output connectivity of the recording sites (Kwan et al., 1987) (e.g., both sites related to shoulder movements, assessed by SMM; for details, see Materials and Methods) (Fig. 3A, third panel from left). Consistent with a recent in vitro study (de la Rocha et al., 2007), NCs increased with the geometric mean of the mean firing rates of the two units (Fig. $3 A$, right). These relationships were qualitatively preserved when possible correlations between the four tested factors were ac-

B
$A$
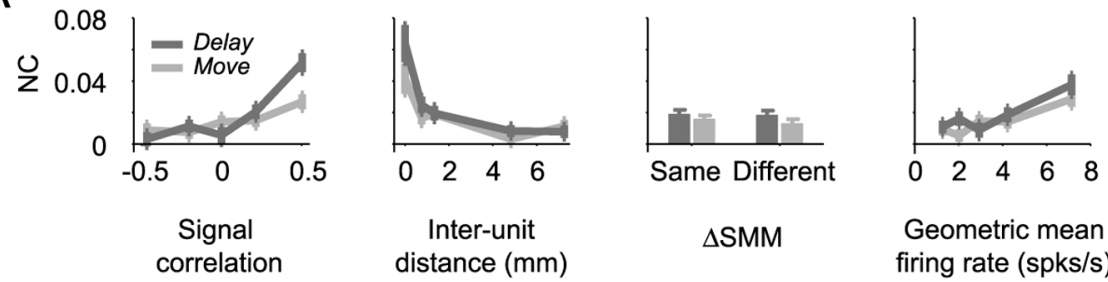

Geometric mean firing rate (spks/s)
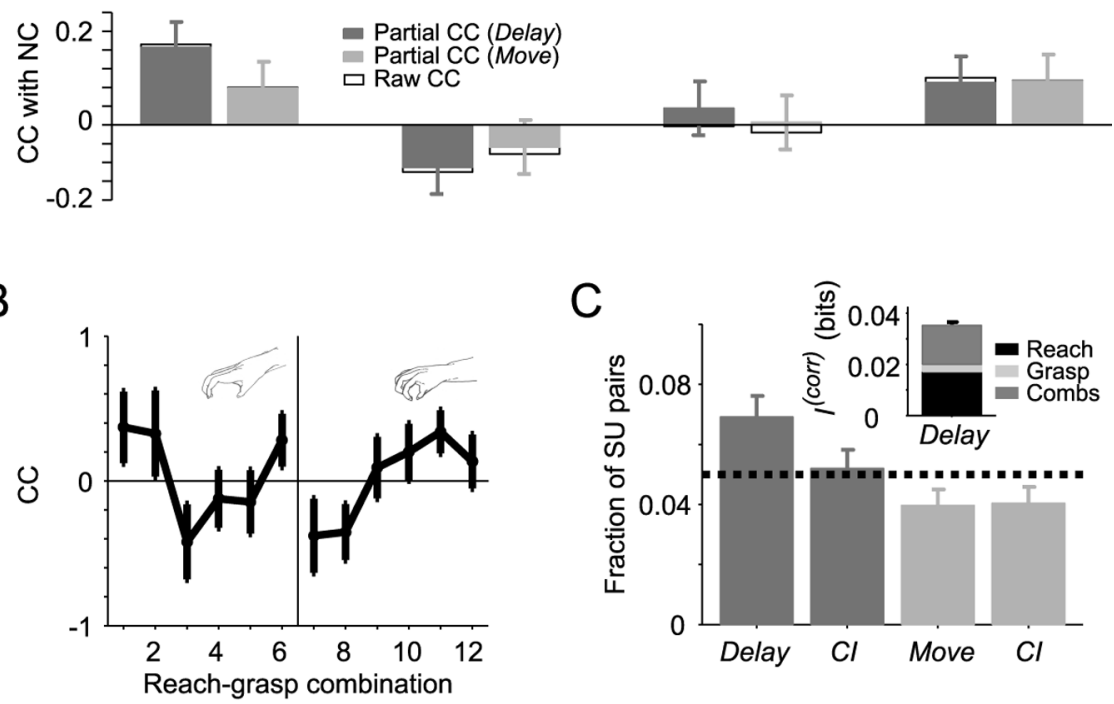

Figure 3. Coarse temporal correlations between premotor SUs during prehension. $\boldsymbol{A}$, Dependence of NCs on other factors. The sample contains 1289 pairs; error bars indicate SEM. Dark and light gray lines correspond to Delay and Move, respectively. Top, Each relevant factor was partitioned into five equal-sized bins, and NCs were averaged over all pairs in that bin. Exceptions include the following: interunit distance, 85 same-electrode pairs and four equal-sized bins; $\Delta$ SMM (difference in sensory-motor mappings), same, 768 pairs; different, 521 pairs. Bottom, Bars show multivariate analysis results, accounting for possible correlations between the four tested factors (partial C(s); open black boxes show the C(s corresponding to the univariate plots from the top. Error bars indicate $99 \%$ confidence intervals for the partial CCs. NCs increase with signal correlations and firing rate and decrease with physical distance between the units. $\boldsymbol{B}$, Example of informative correlations. CCs between two SUs (SU1 is the same as in Fig. $2 A$ ) for trials in different reach-grasp combinations during Delay (1-6, finger opposition grip; 7-12, precision grip; 1, 7, right reach; 2,8 , top right; 3, 9, top left; 4, 10, left; 5, 11, bottom left; 6 , 12, bottom right). Error bars indicate SEM. C(s vary with reach direction in a different manner for the two grasp types. C, Fraction of SU pairs with informative correlations ( $/{ }^{\text {(corr) }}$ significantly above 0; see Materials and Methods). There is a small excess of informative pairs during Delay but not during Move. Inset, Distribution of MI in informative pairs. More than one-third of the MI is about reach-grasp combinations. D, Relationship between CCs and firing rates. Left, Firing rates of the SUs whose CCs are shown in $\boldsymbol{B}$. Right, CCs versus the geometric mean of the firing rates of the two units. CCs depend on the mean rates.

counted for using a multiple linear regression model (Fig. $3 \mathrm{~A}$, bottom).

Coarse temporal correlations varied with reach and grasp parameters. Figure $3 B$ shows the $C C$ at each reach-grasp combination for an SU pair with typical NC ( 0.03 ; the NC is the mean of all 12 CCs, weighted by the number of trials in each combination). During finger opposition trials, CCs were highest when the up- 
A

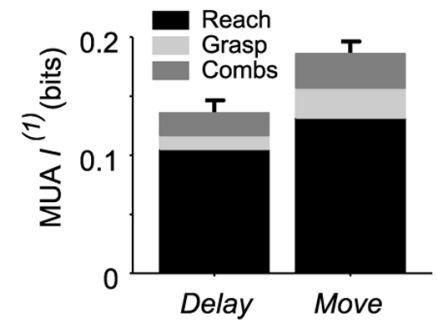

B

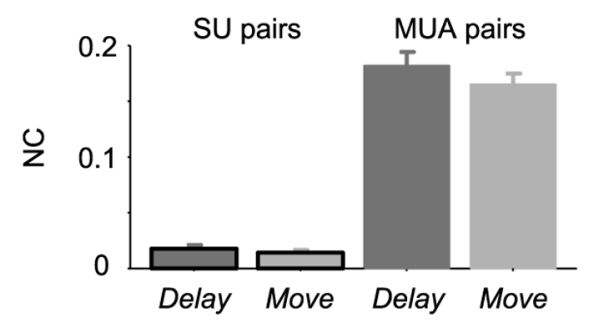

C
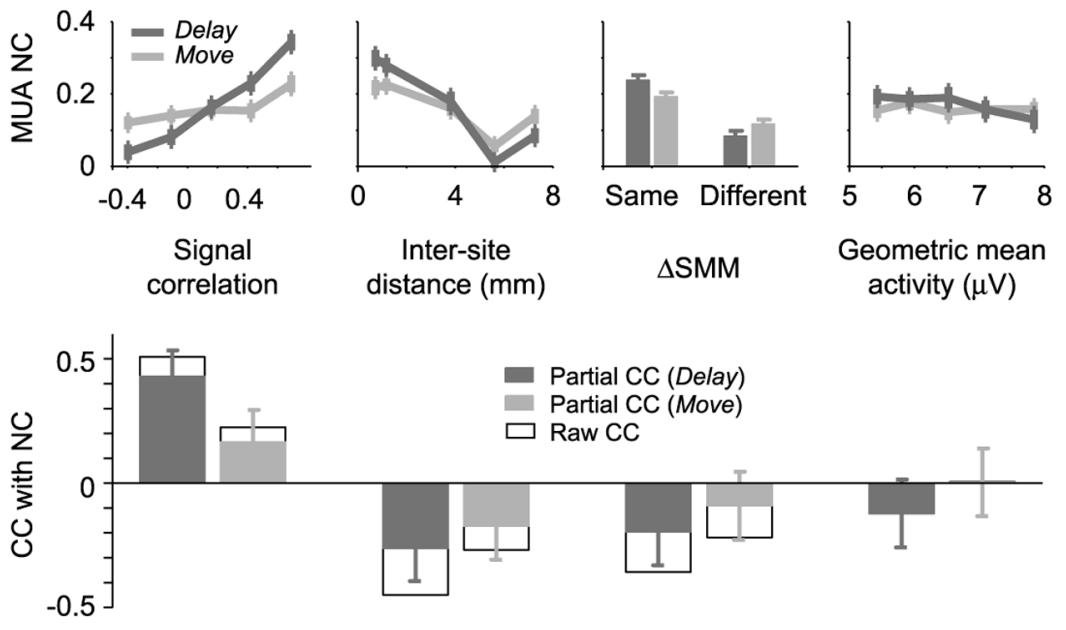

D
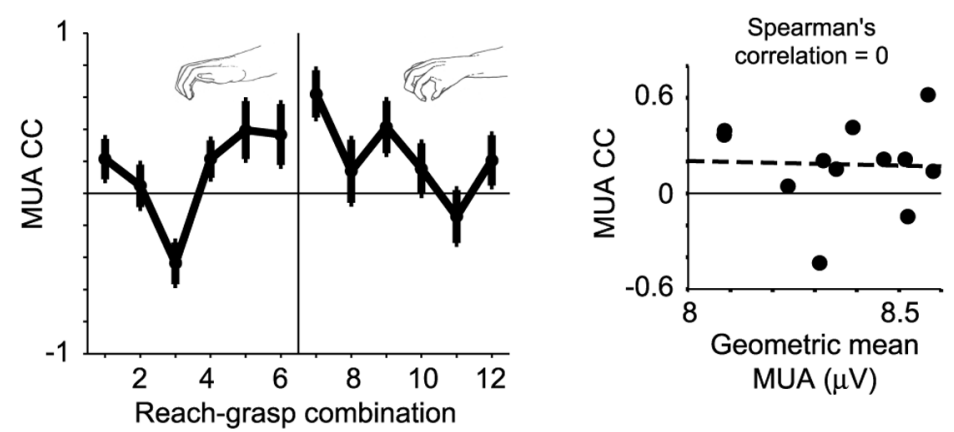

E

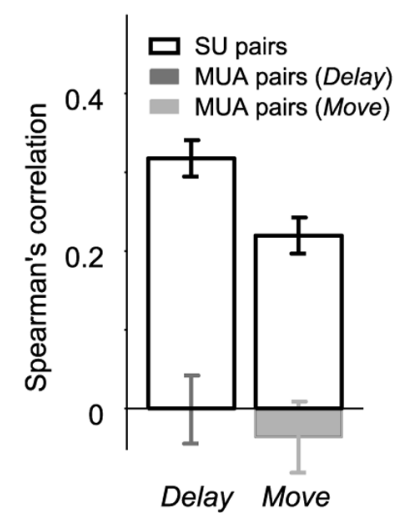

$\mathrm{F}$

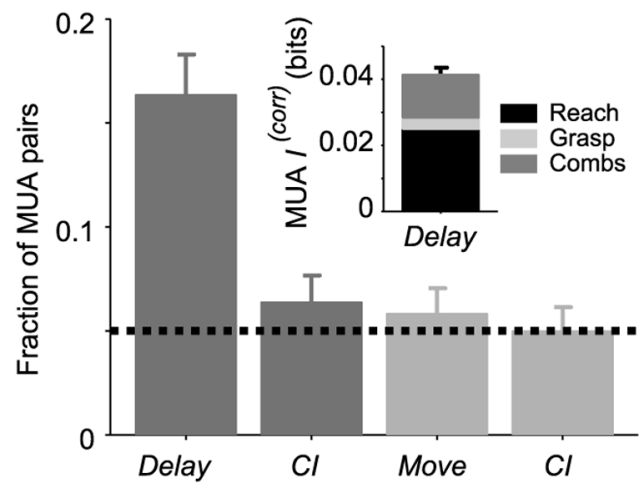

Figure 4. Information in premotor MUAs and in coarse temporal correlations between MUAs. $\boldsymbol{A}$, Information in individual MUAs, averaged over all informative premotor MUAs (Delay, 109 of 142 MUAs; Move, 134 of 142 MUAs). As for SUs, most MI is about reach direction. $\boldsymbol{B}$, Average NC between SUs (left) and MUAs (right). The sample contains 1289 SU pairs and 361 MUA pairs. Open black boxes, NCs between SUs, processed in the same manner as the MUA signal (see Results). NCs between MUAs are an order of magnitude higher than those between SUs. C, Dependence of MUA NCS on other factors. There are 206 same and 155 coming reach direction was to the right, whereas, during precision grip trials, CCs were highest when reaching was to the bottom left. Thus, CCs varied with reach direction and in a different manner for the two grasp types. To quantify reach-grasp coding by coarse temporal correlations, we again used MI. Specifically during Delay, CCs between 89 of 1289 (6.9\%) of the pairs were informative ( $I^{\text {(corr) }}$ significantly above 0; see Materials and Methods), a number higher than chance (binomial test, $p=0.0017$ ) (Fig. 3C). The informative pairs consisted of 115 SUs recorded from 84 different sites; 83 of 89 (93\%) of the pairs were recorded from distinct sites. The fraction of pairs with informative CCs was 6 of $85(7 \%)$ for same-electrode pairs and 83 of $1204(6.9 \%)$ for differentelectrode pairs and thus did not depend on intersite distance ( $\chi^{2}$ test, $\left.p=0.96\right)$. Delay correlations were informative for some SU pairs recorded in the same premotor region (PMd or PMv) but not for SUs recorded in distinct regions (Table 1, second row). In contrast, during Move, the number of informative SU correlations was at chance level regardless of the recording site.

Because SU correlations were related to firing rates (Fig. $3 A$, right), we further tested whether $I^{\text {(corr) }}$ resulted from informative firing rates as follows. For each pair, we shuffled the trial order of one of the units (for each reach-grasp combination separately; CI shuffling; see Materials and Methods). For the sample of SU correlations during Delay, the number of informative pairs diminished to chance level after CI shuffling, indicating that $I^{\text {(corr) }}$ is not a trivial byproduct of informative individual SUs. For the informative pairs during Delay, $41 \pm 3.2 \%$ (mean \pm SEM) of $I^{\text {(corr) }}$ total was about reach-grasp combinations (Fig. 3C, inset).

\footnotetext{
$\leftarrow$

different SMM pairs. In contrast to SU NCs (Fig. 3A), MUA NCS are higher when SMM is the same but do not depend on the activity of the individual MUAs. D. Example of MUA correlations. Left, Delay epoch CCs between two MUAs, recorded from PMd (SMM, elbow flexion) and PMv (SMM, thumb flexion) sites $5.4 \mathrm{~mm}$ apart. (Cs varied with the memorized reach direction in a different manner for the two grasp types. Right, MUA CCs versus the geometric mean of the individual MUAs. $\boldsymbol{E}$, Rank correlations between individual MUAs and C(s between them (gray bars). For reference, open black boxes show rank correlations between SUs. In contrast to SU correlations, CCS between MUA pairs do not depend on the geometric mean activity. $\boldsymbol{F}$, Information in coarse temporal correlations between MUAs. Conventions are the same as in Figure $3 C$. Similar to but considerably more often than among SU correlations, there is an excess of informative pairs, specifically during Delay.
} 
The observation that NCs vary with mean firing rates (Fig. $3 A$, right) suggests that the variation of CCs with reach-grasp combinations (and hence the information carried by CCs) may result from variation of firing rates with reach-grasp combinations. Indeed, for a given SU pair, CCs were generally higher during reach-grasp combinations in which the firing rates of the two units were higher (Fig. 3D, left). To quantify this monotonic yet possibly nonlinear tendency, we used Spearman's rank correlation, which was 0.31 for the example pair (Fig. 3D, right). Over all pairs during Delay, the median rank correlation between firing rates and CCs was 0.35 (bootstrap test, $p<0.001$ ). During Move, rank correlations were lower but also tended to be positive (median, 0.23; Mann-Whitney $U$ test, $p \ll 0.001)$. Thus, the fact that some CCs are informative does not necessarily imply that they convey information distinct from that in the individual SUs. To directly address this issue, we compared the overall information in the activity of a pair of units (and the interactions between them) with the information in the CI case (that is, without any pairwise interactions; $\Delta I^{(1,2)}$ ) (Eq. 2). The number of pairs with $\Delta I^{(1,2)}$ significantly higher than 0 was at chance level during Delay (51 of 1075 of the informative pairs, $4.7 \%$ ) and Move (45 of 1199 pairs, 3.8\%), indicating that coarse temporal interactions between SUs did not contribute information beyond that existing in the CI code.

\section{Coarse temporal correlations between premotor MUAs carry information about reach-grasp combinations}

The results reported above indicate that coarse temporal correlations between individual neurons carried little information about prehension. Although some Delay CCs did carry information about reach-grasp combinations, the number of informative SU pairs was small, and thus we considered MUA, representing the spiking activity of a local group of neurons (see Materials and Methods). During Delay, 109 of 142 (77\%) of the MUAs were informative about prehension parameters, and more were informative during Move ( 134 of $142,94 \%$; $\chi^{2}$ test, $p \ll 0.001$ ). As for SUs, there were no differences in the fractions of informative MUAs in PMd and PMv (Table 1, fourth row). Also akin to SUs, most of the MI carried by informative MUAs was about single prehension parameters (reach direction or grasp type) and only a small part about their combinations (Fig. 4A). Thus, although informative MUAs were more common than informative SUs (Stark and Abeles, 2007), individual MUAs shared the qualitative properties of SUs.

For pairs of simultaneously recorded MUAs, NCs were biased to positive values (bootstrap tests, $p<0.001$ for both epochs; 361 pairs) (Fig. $4 B$ ) and were an order of magnitude higher than NCs between SUs computed in the same epochs (Fig. $4 B$ ). The same results were obtained when SU spike trains were processed in the same manner as the MUA signals (Fig. 4B, open black boxes), indicating that MUA correlations were not higher simply because of their continuous nature. We assessed the dependency of NCs between MUAs on the same four factors as for SU pairs: the dependencies on signal correlations and anatomical distance between the recording sites carried through and were stronger during Delay (Fig. 4C, left two panels). In contrast to SU pairs, NCs between MUAs recorded from sites with similar SMMs (e.g., both finger related) were higher than when recorded from different SMM sites (e.g., shoulder and finger) (Fig. 4C, third panel from left). This relationship was also stronger during Delay. Also in contrast to SU pairs, NCs did not increase with the geometric mean of the individual MUAs (Fig. $4 C$, right). Furthermore, CCs of a given MUA pair in different reach-grasp combinations did not depend on the activity of the individual MUAs. This was the case for the example MUA pair (Fig. 4D) and for the entire sample of pairs, for which the median rank correlations were 0 and -0.034 during Delay and Move, respectively (bootstrap tests, $p=$ 0.36 and $p=0.9$ ) (Fig. $4 E$ ). Thus, coarse temporal correlations between MUAs were much stronger and in some aspects qualitatively different from SU correlations.

CCs between MUAs varied with prehension parameters (Fig. 4D). During Delay but not during Move, 59 of 361 (16\%) of the MUA pairs had informative correlations ( $I^{(\text {corr })}$ significantly above 0 ), a fraction higher than chance (binomial test, $p \ll$ 0.001) (Fig. $4 F$ ) and higher than the corresponding fraction of informative SU correlations during the same epoch $\left(6.9 \% ; \chi^{2}\right.$ test, $p \ll 0.001)$. The informative MUA pairs consisted of 59 of $142(42 \%)$ of the individual MUAs. Information in MUA correlations was not a result of information in individual MUAs: the fraction of informative pairs diminished to chance level after CI shuffling (Fig. $4 F$ ). To test whether the fraction of informative correlations depended on interelectrode distance, we divided the sample of 361 MUA pairs into "near" and "far" according to the median distance $(3.8 \mathrm{~mm})$. The number of informative correlations was similar in the two sets (near, 27 of 180; far, 32 of $181 ; \chi^{2}$ test, $p=0.49$ ), suggesting that the MI in MUA correlations was not a distance-related effect. As for informative SU correlations, more than one-third of the MI carried by informative MUA correlations during Delay was about reach-grasp combinations (Fig. $4 F$, inset).

To assess the possibility that information in MUA correlations differed from that carried by the individual MUAs, we again used $\Delta I^{(1,2)}$. During Delay, 37 of $335(11 \%)$ of the informative pairs had interactions that carried information beyond the information in the individual MUAs $\left(\Delta I^{(1,2)}\right.$ significantly above 0$)$, and similar results were obtained during Move (binomial tests, $p \ll$ 0.001) (Fig. 5A). These numbers were reduced to chance level after CI shuffling. Moreover, similar results were obtained for 
A

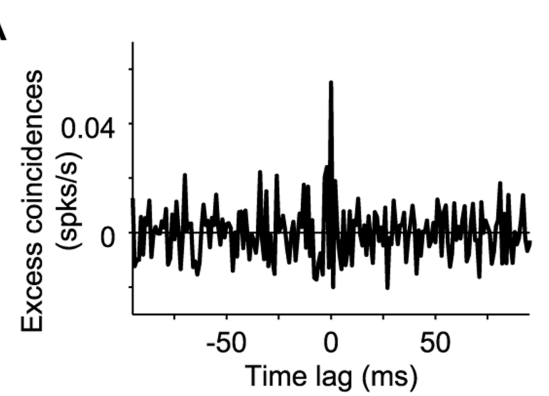

B
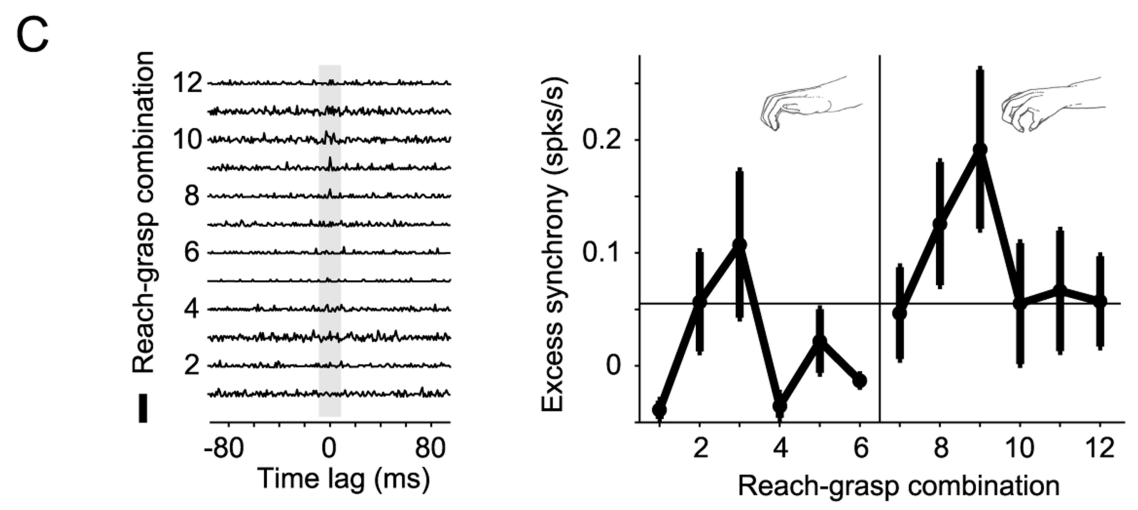

Figure 6. Synchrony between single premotor units. $\boldsymbol{A}, \mathrm{CCH}$ for a pair of PMv units $1.1 \mathrm{~mm}$ apart. The abscissa measures time lag, and the ordinate measures the rate of excess coincidences (see Materials and Methods). The two units were synchronized. $\boldsymbol{B}$, Fraction of precisely correlated SU pairs. The ordinate measures the fraction of pairs that had an above-chance number of coincidences during Delay. The sample size is 944 SU pairs. There is a small excess of precisely synchronized pairs. C, Example of tuned SU synchrony (same pair as in A). Left, CCHs are plotted for each reach-grasp combination. Calibration: 0.25 spikes/s. Right, Excess synchrony rate; error bars indicate SEM; horizontal thin line, mean excess synchrony rate. The excess synchrony rate varies with reach- grasp combination.

MUA pairs recorded in the same and in distinct premotor regions (Table 1, last row). Finally, an equivalent analysis of muscle activity yielded chance level results (Move epoch, 4 of 150 muscle pairs with $\Delta I^{(1,2)}$ significantly above $\left.0 ; p=0.95\right)$.

To study the source of the information in pairwise interactions between MUAs, we compared the MI in MUA correlations $\left(I^{(\text {corr })}\right)$ and in pairwise interactions $\left(\Delta I^{(1,2)}\right)$. Over all MUA pairs, the two measures were positively correlated (Spearman's rank correlation, 0.12 ; bootstrap test, $p=0.012$ ). Focusing on pairs for which both measures were significantly positive, $\Delta I^{(1,2)}$ was consistently higher than $I^{\text {(corr) }}$ (median ratio over 13 pairs, 1.4; Mann-Whitney $U$ test, $p=0.0027$ ). Thus, interactions between MUA pairs carried information beyond that carried by correlations per se.

To determine the contribution of interactions to the overall pairwise information, we measured the fraction $f \Delta I^{(1,2)}$, which could be positive or negative (see Materials and Methods). Over all 361 MUA pairs, $f \Delta I^{(1,2)}$ was biased to positive values during Delay but not during Move (bootstrap tests, $p=0.015$ and $p=$ 0.79 , respectively) (Fig. $5 B$, dashed lines). This did not result from a biased test: for CI shuffled data, $f \Delta I^{(1,2)}$ was $-0.0025 \pm 0.0071$ (mean \pm SEM). For the pairs with $\Delta I^{(1,2)}$ significantly above 0 , Delay $f \Delta I^{(1,2)}$ values were generally higher than during Move ( $U$ test, $p=0.014$ ) (Fig. $5 B$, open black boxes). Although MI in coarse temporal interactions contributed to reach, grasp, and combination coding, contributions during Delay varied with the parameter (nonparametric ANOVA, $p=0.046$ ) (Fig. 5B, grayscale bars). Specifically, the largest contribution of pairwise interactions to the overall pairwise MI was to the coding of reach- grasp combinations (post hoc paired Wilcoxon's signed-rank test comparing contributions to reach and combinations, $p=0.026)$. Qualitatively similar results were obtained during Move (Fig. 5B, right). Thus, coarse temporal correlations between MUA pairs were generally independent of the individual MUAs and contributed information to pairwise codes, the largest contribution being to the coding of reach-grasp combinations.

\section{Synchrony between SUs is temporally precise but carries little information} Up to now, we have focused on neural activity measured at a coarse temporal resolution (400 ms epochs), but, because the nervous system clearly possesses means of processing information much faster, we now turn to correlations measured at a millisecond resolution. Figure $6 A$ shows a Delay epoch excess-coincidence $\mathrm{CCH}$ (see Materials and Methods) for two SUs recorded from PMv sites $1.1 \mathrm{~mm}$ apart. The $\mathrm{CCH}$ exhibited a sharp peak at zero lag, indicating that the units were synchronized: there were 43 coincident spikes, whereas the number expected assuming spike timing does not matter up to $\pm 5 \mathrm{~ms}$ was 17.7 (Poisson test with continuity correction, $p \ll 0.001)$. Of 944 SU pairs recorded by different electrodes, $74(7.8 \%)$ were synchronized (binomial test, $p<$ 0.001) (Fig. 6B). A smaller but above chance number of synchronized pairs was observed during Move $(6.5 \% ; p=0.027)$. Thus, some pairs of premotor units recorded from different sites were precisely synchronized, mainly during memorization.

Despite its low rate, excess synchrony might carry information about prehension. For the example pair, the excess synchrony varied with reach and grasp and had $I^{\text {(corr) }}$ of 0.12 bits (Fig. $6 C$ ). Thus, synchronous interactions between pairs of SUs may carry information about behavior (Dan et al., 1998; Hatsopoulos et al., 1998). However, even the excess synchrony is influenced by firing rates. To see this, consider a pair of neurons with fixed interconnections: when both are silent, excess synchrony is always 0 . To obtain an estimate of MI in excess synchrony that is not hindered by firing rates, we again used CI shuffling: for the example pair, the mean $I^{\text {(corr) }}$ obtained after CI shuffling was 0.015 , and the nonshuffled $I^{\text {(corr) }}$ was larger than 991 of 1000 of the shuffled values $(p<0.01)$. Thus, the information carried by the excess synchrony of this specific pair did not result from information in the firing rates. However, overall, the excess synchrony of only 48 of 944 (5.1\%; binomial test, $p=0.47)$ pairs carried more information than after CI shuffling (Delay; a similar result was obtained during Move). Thus, although synchrony between SU pairs occurred more frequently than expected if spike timing did not matter, it rarely carried information beyond that carried by firing rates.

Synchronous interactions between MUAs are common, strong, and sometimes carry information about prehension Synchronous interactions were a prominent feature of precise temporal correlations between pairs of simultaneously recorded 
MUAs (Fig. 7A). During Delay, 124 of 361 (34\%) of the MUA pairs were synchronized (binomial test, $p \ll 0.001$ ) (Fig. $7 B$ ). Most individual MUAs (118 of 142, 83\%) participated in synchronized pairs. A similar result was obtained during Move (134 of 361 pairs, $37 \% ; \chi^{2}$ test, $\left.p=0.44\right)$. We did not observe oscillatory MUAs or oscillatory CCHs between MUAs as seen for unsorted spikes during visual stimulation (Eckhorn et al., 1988; Gray et al., 1989; Womelsdorf et al., 2007). In contrast to a weak distance dependency of SU synchrony (Spearman's rank correlation, $-0.031 ; p=0.19)$, synchrony was higher for MUAs recorded from closer sites (rank correlation, -0.63 ; bootstrap test, $p<$ 0.001) (Fig. 7C).

Synchrony was higher for MUA pairs with higher coarse temporal correlation (Spearman's rank correlation between coarse and precise temporal CCs, 0.53 ; bootstrap test, $p<0.001)$. Compared with SU pairs, peak half-widths of CCHs between MUA pairs were typically larger (median, 6 $\mathrm{ms}$ ), but this was entirely attributable to the processing of the MUA signals (bandpass and low-pass filtering; see Materials and Methods). Synchrony was more common among MUA pairs than among SU pairs (Fig. 7D), and the synchrony signal-to-noise ratio (SNR) (defined here as the $\mathrm{CCH}$ value at zero lag divided by the SD over all lags excluding the three central lags) was larger for MUA pairs than for SU pairs ( $U$ test, $p$ $\ll 0.001$ ) (Fig. 7E). The same result was obtained for the subset of synchronized pairs (median SNRs, 2.3 for SUs and 3.3 for MUAs; $p \ll 0.001)$. Thus, SU and MUA precise temporal correlations shared a tendency to occur at zero lag and showed a distance dependency, but MUA synchrony was considerably more common and stronger than SU synchrony.

Synchrony between some MUA pairs varied with prehension; an example is shown in Figure $7 F$ (same pair as in Fig. $7 A$ ). The information carried by the example pair was 0.071 bits, higher than in all CI shuffled cases $(p<0.001)$ and indicating that the information was carried by synchrony per se. During Delay, the synchrony of 25 of 361 (7\%) of the MUA pairs was informative (binomial test, $p=0.065$ ). MUA pairs with informative coarse temporal correlations did not necessarily have informative synchrony (Spearman's rank correlation, -0.095 ; bootstrap test, $p=$ 0.95). However, as for the coarse temporal correlations, $41 \pm$ $4.3 \%$ of the MI carried by informative MUA synchrony was about reach-grasp combinations (Fig. $7 G$ ). Similar results were obtained during Move, and the total number of informative synchronous interactions was 50 of 722 (7\%; binomial test, $p=$ 0.014). In summary, synchronous interactions between MUA pairs were ubiquitous, strong, and, in some cases, carried information about reach-grasp combinations underlying prehension.

\section{Discussion}

Two issues complicate the study of information coded by interactions between individual neurons. First, correlations between
B

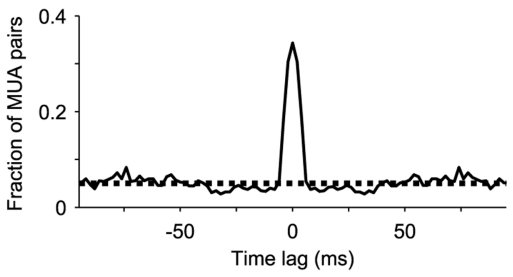

E

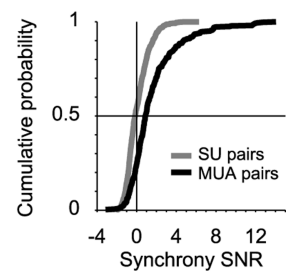

G

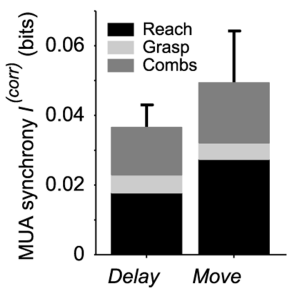

Figure 7. Synchrony between premotor MUAs. $A$, Example of a $C \mathrm{CH}$ for a synchronized pair of MUAs. The recording electrodes different manner for the two grasp types. $\mathbf{G}$, Information in synchrony between MUAs. There is an excess of informative synchronous interactions, and more than one-third of the Ml carried by informative synchrony is about reach-grasp combinations.

individual neurons are typically weak, particularly for nonneighboring neurons (Ts'o et al., 1986; Hatsopoulos et al., 1998; Sakurai and Takahashi, 2006). Second, correlations between spiking of individual neurons depend on their firing rates and other SU properties (de la Rocha et al., 2007; Tetzlaff et al., 2008). Here we showed that multiunit correlations are typically stronger than SU correlations (deCharms and Merzenich, 1996; Supèr and Roelfsema, 2005), essentially independent of the activity of the individual MUAs, and carry additional information.

To see how MUA correlations can be more common than SU correlations, consider that a fraction $f$ of the neurons in two neuronal groups has similar activity. Random selection of one neuron from each group yields an $f^{2}$ chance to observe correlated SU activity, whereas the two MUAs are always correlated. Furthermore, strong MUA correlations may result from weak SU correlations because, when multiple SU pairs are considered simultaneously, correlations are amplified because of signal-to-noise improvement (Bedenbaugh and Gerstein, 1997; Zeitler et al., 2006).

Strong correlations between MUAs do not necessarily imply independence from the individual MUAs, but, because MUA reflects multineuronal spiking, the neurons contributing the most to one MUA are not necessarily those contributing the most to the correlation with another. Thus, MUA correlations and individual MUAs can carry distinct information. Because neu- 
rons are particularly sensitive to correlated input (Abeles, 1982b; Rudolph and Destexhe, 2001), the distinct information carried by MUA correlations can be transmitted to other cortical targets given sufficient connectivity between neuronal groups. Although motor cortex corticocortical connections are spread over several millimeters (Huntley and Jones, 1991; Dum and Strick, 2005), an all-to-all connectivity is not necessary because information can be transmitted stepwise.

In the transition from spiking activity of all local neurons to MUA, substantial information is lost and correlation patterns specific to some neuronal pairs are obscured; some can be revealed by analyzing SU pairs. Whereas not all neurons contributing to the MUA signal can be recorded using present technologies, MUA recordings are relatively easy to obtain and stable over time (Supèr and Roelfsema, 2005; Stark and Abeles, 2007).

\section{Contribution of pairwise interactions to neural coding}

The mere existence of correlations between neuronal groups does not indicate that correlations carry information. Only if SU interactions contributing to MUA interactions are informative will the latter be informative. Previous studies have yielded mixed results in this respect. Some showed that individual cortical neurons are independent or redundant encoders (Gawne and Richmond, 1993; Reich et al., 2001), whereas others emphasized that correlations vary between behavioral states and might carry information (Gray et al., 1989; Vaadia et al., 1995; Riehle et al., 1997; Steinmetz et al., 2000; Hatsopoulos et al., 2003). However, only a few studies have shown explicitly that interneuronal correlations contribute information beyond the single-neuron codes (Dan et al., 1998; Maynard et al., 1999; Romo et al., 2003). The current work extends the latter results by showing that interactions between neurons, and in particular between neuronal groups, vary between reach-grasp behaviors and carry information about them. Nonredundant information carried by pairwise interactions may arise from signal and noise correlations with opposing signs, from behavioral modulation of noise correlations, and/or from modulations of higher-than-second-order statistics of the pairwise activity. We showed that information in pairwise interactions is not solely attributable to behavioral modulations of linear correlations, but a systematic study of these sources awaits future work.

In previous reports, when correlations did vary with behavior and were thus potentially informative, information was mainly about the same parameter encoded by the individual neurons (deCharms and Merzenich, 1996; Hatsopoulos et al., 1998). Exceptions are visual cortex correlations, which may be specific to global stimulus features (Ts'o et al., 1986; Gray et al., 1989). Here, information carried by neuronal interactions differed qualitatively from information carried by individual channels (SUs or MUAs): whereas most single-channel information was about reach direction or grasp type, the largest contribution of informative MUA interactions was to the coding of reach-grasp combinations.

Coarse and precise temporal correlations were typically correlated for a given MUA pair, but the information carried by these two forms of correlation was distinct. Thus, although coarse and precise temporal correlations may result from common mechanisms, they can form independent information-carrying channels.

Even during memorization, only one-sixth of MUA correlations were informative, and less than one-half of the MUAs participated in informative correlations. This relatively small fraction might suggest that pairwise interactions contribute little to neural coding. However, several points suggest otherwise. First, the number of pairs and their potential information-carrying capacity grows with the square of the number of channels. Second, pairwise interactions coded information that differed qualitatively from single-channel information. Third, the information in a neural code depends on the code itself but also on the specific experimental paradigm. Thus, combination-specific information in pairwise interactions may be higher in experiments requiring the simultaneous internal representation of multiple combinations followed by action on one (Triesch and von der Malsburg, 1996). The greater the number and complexity of the internal representations, the larger the information accrued by considering interneuronal interactions is expected to be.

\section{Specific correlation patterns}

Some of the observed correlation patterns deserve attention. First, correlations varied considerably with interneuronal distance, extending previous findings (Vaadia et al., 1995; Hatsopoulos et al., 1998; Sakurai and Takahashi, 2006) and indicating that properties of correlations between distant neurons should not be inferred from studying correlations between neurons recorded by the same microelectrode. Second, we did not observe oscillatory MUAs or synchronous oscillations between MUAs. This differs from local-field potentials (LFPs) measured in the motor system that typically exhibit beta-range oscillations (Murthy and Fetz, 1992; Baker et al., 1999; Rubino et al., 2006) and from visual system MUAs that oscillate in the gamma range (Eckhorn et al., 1988; Kreiter and Singer, 1996; Womelsdorf et al., 2007). Although some differences might be attributed to experimental technicalities, it is possible that different mechanisms underlie coherent cortical activity in frontal versus occipital cortices and/or during sensory stimulation versus motor memory. Third, most precise temporal correlations between MUAs were positive. Although SU CCHs are more sensitive to excitatory than inhibitory interactions (Aertsen and Gerstein, 1985), MUAs should suffer much less from a floor effect. However, because most intracortical connections are excitatory $(\sim 85 \%)$ (Braitenberg and Schuz, 1991), negative SU correlations are obscured despite the typically larger inhibitory postsynaptic potentials. Finally, most precise temporal SU correlations were synchronous interactions, apparently at odds with the Synfire chain hypothesis (Abeles, 1982a) that predicts an approximately uniform distribution of time lags of excess coincidences. MUA precise temporal correlations were almost exclusively at zero lag, and synchrony between MUAs might be a mark of synchronous coactivation of two chains (Abeles et al., 2004).

\section{Implications for reach-grasp coordination and binding}

Our results show that correlations in premotor cortex are typically weaker and carry less information during movement than during reach-grasp memorization. This general decorrelation may reflect a processing shift, "downloading" composite commands to the primary motor cortex (M1) and/or spinal cord during actual movement. This speculation, namely that during movement, M1/spinal cord correlations are higher than (1) M1/ spinal cord correlations during memorization and (2) premotor correlations during movement is experimentally testable. It is also consistent with the idea that, during movement, muscles form transient synergies facilitating motor control, with the finding that, during movement, correlations between M1 LFPs are generally reduced (Cardoso de Oliveira et al., 2001) and with the finding that M1 neurons correlated with similar muscles tend to synchronize (Jackson et al., 2003). 
Although PMd has been classically related to arm movements and reaching and PMv to hand movements and grasping (Luppino and Rizzolatti, 2000), recent evidence indicates mixed connectivity and coding of both reach and grasp parameters in the two regions (Raos et al., 2004; Dum and Strick, 2005; Stark and Abeles, 2007; Stark et al., 2007). Here, we showed that pairwise interactions between premotor neuronal groups carry information about prehension regardless of the specific recording sites. Thus, rather than forming distinct functional modules, information processing in $\mathrm{PMd}$ and $\mathrm{PMv}$ appears to be highly distributed.

Two prototypical solutions were proposed for the "property binding" problem (Treisman, 1996). One is the "cardinal cell" idea (Barlow, 1972), suggesting that parameter combinations are represented by single-neuron activity. The other is the "correlation theory" (von der Malsburg, 1981), suggesting that parameter combinations are represented by correlations between pairs of neurons. Solutions involving higher-order interactions have also been suggested (Abeles et al., 2004). We found that, although correlations between single neurons may be temporally precise, they are generally weak, so correlation between one pair of neurons might not have functional relevance. In contrast, pairwise interactions and synchrony between neuronal groups are stronger, common, and sometimes informative. The functional relevance of synchronous interactions might be at the population level, at a spatial resolution of a few hundred neurons. Thus, the current results support the possibility that correlations between groups of premotor neurons bind reach and grasp components, forming detailed internal representations of the upcoming movement, prehension.

\section{Appendix}

Here the specifics pertaining to estimating MI between neural activity and reach-grasp behavior are explained. The computational approach is based on direct estimation of MI between neural activity and reach-grasp behavior (Eq. A1). Equivalent results were obtained when estimating MI using a decoding method. In the latter approach, we used statistical classifiers to predict reachgrasp behavior from neural activity (as in the study by Stark and Abeles, 2007) and then estimated the MI between the actual and predicted behaviors.

\section{Information between single-channel activity and reach-grasp behavior}

To determine the MI between different aspects of neural activity (SU spike count or MUA voltage) and motor behavior (memory or action), we denote neural activity by $X$, behavior in general by $B$, reach direction by $R$, and grasp type by $G$. The MI $I(X ; B)$ measures the information the random variable $X$ conveys about another variable $B$. The information is calculated from the joint distribution $p(x, b)$ of the variables $X$ and $B$ and is given in bits as follows (Cover and Thomas, 1991):

$$
I_{\hat{p}}(X ; B)=\sum_{x, b} \hat{p}(x, b) \log _{2} \frac{\hat{p}(x, b)}{\hat{p}(x) \hat{p}(b)} .
$$

The subscript $\hat{p}$ indicates that $I(X ; B)$ is evaluated using the estimated joint distribution $\hat{p}(x, b)$. It is technically difficult to evaluate the joint distribution when a relatively small number of trials are available: the full table $p(x, b)$ is typically sparse in such cases. To see this, consider the discrete (spike count) case, in which there are many combinations of $x$ and $b$ that do not occur at all. To alleviate this difficulty, we quantized neural activity into two (or three, if the mean number of trials per reach-grasp combination was above 25) bins so the number of counts per bin was approximately equal (Pola et al., 2003). According to the data processing inequality (Cover and Thomas, 1991), quantization cannot increase the MI. Because the actual number of trials per reach-grasp combination was above 20 , there were 11 samples (median; ranges: SUs, 7-26; MUAs, 7-17) in each cell of the estimated table $\hat{p}(x, b)$.

Because information is a non-negative measure, it is biased to positive values. We estimated the bias by random shuffling of the behavioral tags $b$ between trials (resampling without replacement), recomputing the information, and averaging over 1000 shuffles. The bias was removed from the estimated information by subtraction. The validity of this approach was assessed using artificial neural activity that carried various amounts of known information about the behavior. We found that the bias estimated using the shuffling procedure is a conservative estimate and has two advantages over, for instance, the first-order approximation suggested by Panzeri and Treves (1996). First, it is readily extended to correcting biased information estimates in any neural code (in particular, in channel pairs), and second, it yields $p$ values for testing the null hypothesis of zero information. Specifically, the probability of obtaining a given MI assuming the null hypothesis was estimated as the fraction of shuffled values larger than the uncorrected MI.

To study prehension and combination coding, we decomposed the total MI into information about reach, grasp, and nonlinear reach-grasp combinations. We thus estimated the following measures using Equation A1:

$$
\begin{aligned}
& I_{\text {total }}^{(1)}=I_{\hat{p}}(X ; R, G), \\
& I_{\text {reach }}^{(1)}=I_{\hat{p}}(X ; R), \\
& I_{\text {grasp }}^{(1)}=I_{\hat{p}}(X ; G),
\end{aligned}
$$

which are, respectively, the MI between neural activity and all 12 behaviors, the set of six reach directions, and the two grasp types. Here and in other notations, the superscript denotes the neural code, so $I^{(1)}$ indicates MI in the activity of one SU or one MUA. All measures were corrected for bias as explained above. A key question in the current study was whether neural activity encodes nonlinear combinations of reach and grasp or whether reach and grasp coding are independent. To obtain a quantitative measure of such coding, we defined the following measure

$$
I_{\mathrm{comb}}^{(1)}=I_{\hat{p}}(R ; G \mid X)-I(R ; G) .
$$

A similar measure was used previously (Adelman et al., 2003). The definition of $I_{\text {comb }}$ includes $I(R ; G)$, a quantity external to the neural code that is subtracted from the conditional MI, $I(R$; $G \mid X)$. For independent $R$ and $G, I(R ; G)=0$ and $I_{\text {comb }}$ is nonnegative. Although the reach and grasp variables were independent by design, there were small empirical deviations, with a median value of $I(R ; G)=0.0018$ bits $\left(\sim 2 \%\right.$ of $I^{(1)}$ total $)$. Thus, the current definition of $I_{\text {comb }}$ (Eq. A3) is slightly conservative; all reported results were qualitatively identical when $I(R ; G)$ was not subtracted.

To prove Equation 1 in Materials and Methods, consider (1) that the chain rule for MI is $I(R, G ; X)=I(R ; X)+I(G ; X \mid R)$ and (2) that conditional MI can be written as a difference of conditional entropies, $I(G ; X \mid R)=H(G \mid R)-H(G \mid X, R)$ (Cover and Thomas, 1991). Plugging 2 into 1 and adding $I(G ; X)-H(G)+$ 
$H(G \mid X)=0$ (the definition of MI) to the right side, we obtain $I(R, G ; X)=I(R ; X)+H(G \mid R)-H(G \mid X, R)+I(G ; X)-H(G)$ $+H(G \mid X)$. Collecting terms, we obtain $I(R, G ; X)=I(R ; X)+$ $I(G ; X)+I(R ; G \mid X)-I(R ; G)$, which can be rewritten as $I_{\text {total }}=$ $I_{\text {reach }}+I_{\text {grasp }}+I_{\text {comb }}$ (Eq. 1). The latter expression is therefore an exact decomposition of $I_{\text {total }}$, which applies to any neural code including temporal correlations.

Computationally, $I_{\text {comb }}$ can thus be estimated in two mathematically equivalent ways: by the definition (Eq. A3) or by a difference (from Eq. 1). Because the two calculation methods involve different terms, there may be numerical differences in the outcome as bias was removed separately for each term; however, identical results were obtained for the two methods.

\section{Information between two-channel correlations and behavior}

To measure information in coarse temporal correlations per se, without any consideration of the information in the individual channels (SUs or MUAs), we repeated the procedure explained above after substituting the single-trial neural activity (SU spike count or MUA voltage) $x_{i}(t)$ by a single-trial pairwise measure. For MUAs, we used the $Z$-score product $z_{i j}(t)$ as defined in Materials and Methods. However, the spike count is a discrete random variable, and, as long as spike count distributions are not identical for all reach-grasp combinations, computing a $Z$-score injects (adds false) information into them. To avoid this, we added a small non-integer number (randomly and uniformly distributed between -0.5 and 0.5 ) to each spike count before standardization. We verified that this continuity correction resulted in unbiased estimates of MI in pairwise correlations in two ways: by generating artificial spike trains with no relation to behavior (independent Poisson processes) and by computing the information in $z_{i j}(t)$ values of real SUs for which the match between neural activity and reach-grasp behavior was randomly shuffled (mixing test). In contrast to the raw $z_{i j}(t)$ values, the continuity-corrected $z_{i j}(t)$ values contained no information in either test.

For precise temporal correlations, $x_{i}(t)$ was replaced by the trial-specific excess synchrony rate (for SU pairs) or by the trialspecific zero-lag CC (for MUA pairs). Thus, MI was measured between reach-grasp behavior and the pairwise correlation between two channels on a trial-to-trial basis. We denote the total and decomposed information in the correlations between two channels by $I_{\text {total }}^{(\text {corr })}$ and by $I_{\text {reach }}^{(\text {corr }} I_{\text {grasp }}^{\text {(corr) }} I_{\text {comb }}^{\text {(corr) }}$ respectively.

\section{Overall information}

To quantify the overall information in the activity of two channels (SUs or MUAs) about behavior, namely the information in the two individual channels and in the pairwise interactions between them (including correlations) $I^{(1,2)}$, we first estimated the joint distribution of the quantized pairwise neural activity with behavior, $\hat{p}\left(x_{1}, x_{2}, b\right) . x_{1}$ and $x_{2}$ denote neural activity in the two channels, and $b$ can represent either reach direction $r$, grasp type $g$, or the full behavior $(r, g)$. We then estimated the information between the combined pairwise activity and behavior using the following expression:

$$
I_{\hat{p}}^{(1,2)}\left(X_{1}, X_{2} ; B\right)=\sum_{x_{1}, x_{2}, b} \hat{p}\left(x_{1}, x_{2}, b\right) \log _{2} \frac{\hat{p}\left(x_{1}, x_{2}, b\right)}{\hat{p}\left(x_{1}, x_{2}\right) \hat{p}(b)} .
$$

As for individual channels, we decomposed the information in the pairwise activity:

$$
\begin{aligned}
& I_{\text {total }}^{(1,2)}=I_{\hat{p}}\left(X_{1}, X_{2} ; R, G\right), \\
& I_{\text {reach }}^{(1,2)}=I_{\hat{p}}\left(X_{1}, X_{2} ; R\right), \\
& I_{\text {grasp }}^{(1,2)}=I_{\hat{p}}\left(X_{1}, X_{2} ; G\right), \\
& I_{\text {comb }}^{(1,2)}=I_{\hat{p}}\left(R ; G \mid X_{1}, X_{2}\right)-I(R ; G) .
\end{aligned}
$$

To measure the information in pairwise activity that was contributed specifically by pairwise interactions between the two single channels, we compared the information provided by the joint activity of the two channels with a reference. Here, the reference is the MI provided when the channels encode behavior independently (the CI code; Eq. A6). An alternative way to quantify this notion is a synergy/redundancy measure (Gawne and Richmond, 1993), SynRed $\left(X_{1}, X_{2}\right) \equiv I\left(X_{1}, X_{2} ; B\right)-I\left(X_{1} ; B\right)-I\left(X_{2} ; B\right)$, in which the reference is essentially the sum of single-channel information. However, such a measure does not quantify the excess/ redundant information in the joint code per se because it is influenced by the similarity between the mean activity patterns of the individual channels (signal correlations). To see this, consider two neurons that have identical mean activity patterns each carrying 1 bit of information about reach direction. Assume that these neurons encode additional independent information by their joint activity about grasp type, amounting to 1 bit. SynRed $\left(X_{1}, X_{2}\right)$ in this case is 0 , despite the independent information in the joint code. We thus consider as a reference the distribution in which the channels are independent given the behavior (CI):

$$
\hat{p}^{\mathrm{CI}}\left(x_{1}, x_{2}, b\right)=\hat{p}\left(x_{1} \mid b\right) \hat{p}\left(x_{2} \mid b\right) p(b) .
$$

The information in the CI distribution about different aspects of behavior, denoted by $I_{\text {total }}^{(1,2) \mathrm{CI}} I_{\text {reach }}^{(1,2) \mathrm{CI}}$ and so on, is computed as in Equations A4 and A5, with $\hat{p}^{\mathrm{CI}}$ substituting for $\hat{p}$. Another way to estimate $I^{(1,2) \mathrm{CI}}$ is empirically by actually shuffling the data (using 1000 CI shuffles; see Materials and Methods). The analytical (Eq. A6) and empirical (shuffling) methods yielded nearly identical results (Spearman's rank correlation for the sample of 361 MUA pairs, 0.93), and thus results are reported for the analytical method.

\section{References}

Abeles M (1982a) Local cortical circuits: an electrophysiological study. Berlin: Springer.

Abeles M (1982b) Role of the cortical neuron: integrator or coincidence detector? Isr J Med Sci 18:83-92.

Abeles M, Gat I (2001) Detecting precise firing sequences in experimental data. J Neurosci Methods [Erratum (2001) 112:203] 107:141-154.

Abeles M, Hayon G, Lehmann D (2004) Modeling compositionality by dynamic binding of synfire chains. J Comput Neurosci 17:179-201.

Adelman TL, Bialek W, Olberg RM (2003) The information content of receptive fields. Neuron 40:823-833.

Aertsen AM, Gerstein GL (1985) Evaluation of neuronal connectivity: sensitivity of cross-correlation. Brain Res 340:341-354.

Baker SN, Kilner JM, Pinches EM, Lemon RN (1999) The role of synchrony and oscillations in the motor output. Exp Brain Res 128:109-117.

Barlow HB (1972) Single units and sensation: a neuron doctrine for perceptual psychology? Perception 1:371-394.

Bedenbaugh P, Gerstein GL (1997) Multiunit normalized cross correlation differs from the average single-unit normalized correlation. Neural Comput 9:1265-1275.

Braitenberg V, Schuz A (1991) Anatomy of the cortex. Berlin: Springer.

Cardoso de Oliveira S, Gribova A, Donchin O, Bergman H, Vaadia E (2001) Neural interactions between motor cortical hemispheres during bimanual and unimanual arm movements. Eur J Neurosci 14:1881-1896.

Cover TM, Thomas JA (1991) Elements of information theory. New York: Wiley-Interscience. 
Dan Y, Alonso JM, Usrey WM, Reid RC (1998) Coding of visual information by precisely correlated spikes in the lateral geniculate nucleus. Nat Neurosci 1:501-507.

Dum RP, Strick PL (2005) Frontal lobe inputs to the digit representations of the motor areas on the lateral surface of the hemisphere. J Neurosci 25:1375-1386.

deCharms RC, Merzenich MM (1996) Primary cortical representation of sounds by the coordination of action-potential timing. Nature 381:610-613.

de la Rocha J, Doiron B, Shea-Brown E, Josić K, Reyes A (2007) Correlation between neural spike trains increases with firing rate. Nature 448:802-806.

Eckhorn R, Bauer R, Jordan W, Brosch M, Kruse W, Munk M, Reitboeck H] (1988) Coherent oscillations: a mechanism of feature linking in the visual cortex? Multiple electrode and correlation analyses in the cat. Biol Cybern 60:121-130.

Gawne TJ, Richmond BJ (1993) How independent are the messages carried by adjacent inferior temporal cortical neurons? J Neurosci 13:2758-2771.

Gray CM, König P, Engel AK, Singer W (1989) Oscillatory responses in cat visual cortex exhibit inter-columnar synchronization which reflects global stimulus properties. Nature 338:334-337.

Grover FS, Buchwald JS (1970) Correlation of cell size with amplitude of background fast activity in specific brain nuclei. J Neurophysiol 33:160-171.

Hatsopoulos NG, Ojakangas CL, Paninski L, Donoghue JP (1998) Information about movement direction obtained from synchronous activity of motor cortical neurons. Proc Natl Acad Sci USA 95:15706-15711.

Hatsopoulos NG, Paninski L, Donoghue JP (2003) Sequential movement representations based on correlated neuronal activity. Exp Brain Res 149:478-486.

Hoffman KL, McNaughton BL (2002) Coordinated reactivation of distributed memory traces in primate neocortex. Science 297:2070-2073.

Huntley GW, Jones EG (1991) Relationship of intrinsic connections to forelimb movement representations in monkey motor cortex: a correlative anatomic and physiological study. J Neurophysiol 66:390-413.

Jackson A, Gee VJ, Baker SN, Lemon RN (2003) Synchrony between neurons with similar muscle fields in monkey motor cortex. Neuron 38:115-125.

Kilner JM, Baker SN, Lemon RN (2002) A novel algorithm to remove electrical cross-talk between surface EMG recordings and its application to the measurement of short-term synchronisation in humans. J Physiol 538:919-930

Kohn A, Smith MA (2005) Stimulus dependence of neuronal correlation in primary visual cortex of the macaque. J Neurosci 25:3661-3673.

Kreiter AK, Singer W (1996) Stimulus-dependent synchronization of neuronal responses in the visual cortex of the awake macaque monkey. J Neurosci 16:2381-2396.

Kwan HC, Murphy JT, Wong YC (1987) Interaction between neurons in precentral cortical zones controlling different joints. Brain Res 400:259-269.

Laurent G, Davidowitz H (1994) Encoding of olfactory information with oscillating neural assemblies. Science 265:1872-1875.

Luppino G, Rizzolatti G (2000) The organization of the frontal motor cortex. News Physiol Sci 15:219-224.

Lee D, Port NL, Kruse W, Georgopoulos AP (1998) Variability and correlated noise in the discharge of neurons in motor and parietal areas of the primate cortex. J Neurosci 18:1161-1170.

Maynard EM, Hatsopoulos NG, Ojakangas CL, Acuna BD, Sanes JN, Normann RA, Donoghue JP (1999) Neuronal interactions improve cortical population coding of movement direction. J Neurosci 19:8083-8093.

Murthy VN, Fetz EE (1992) Coherent 25- to 35-Hz oscillations in the sensorimotor cortex of awake behaving monkeys. Proc Natl Acad Sci USA 89:5670-5674.

Nirenberg S, Carcieri SM, Jacobs AL, Latham PE (2001) Retinal ganglion cells act largely as independent encoders. Nature 411:698-701.

Paninski L, Fellows MR, Hatsopoulos NG, Donoghue JP (2004) Spatiotemporal tuning of motor cortical neurons for hand position and velocity. J Neurophysiol 91:515-532.
Panzeri S, Treves A (1996) Analytical estimates of limited sampling biases in different information measures. Network 7:87-107.

Pola G, Thiele A, Hoffmann KP, Panzeri S (2003) An exact method to quantify the information transmitted by different mechanisms of correlational coding. Network 14:35-60.

Raos V, Umiltá MA, Gallese V, Fogassi L (2004) Functional properties of grasping-related neurons in the dorsal premotor area F2 of the macaque monkey. J Neurophysiol 92:1990-2002.

Reich DS, Mechler F, Victor JD (2001) Independent and redundant information in nearby cortical neurons. Science 294:2566-2568.

Riehle A, Grün S, Diesmann M, Aertsen A (1997) Spike synchronization and rate modulation differentially involved in motor cortical function. Science 278:1950-1953.

Romo R, Hernández A, Zainos A, Salinas E (2003) Correlated neuronal discharges that increase coding efficiency during perceptual discrimination. Neuron 38:649-657.

Roskies AL (1999) The binding problem. Neuron 24:7-9, 111-125.

Rubino D, Robbins KA, Hatsopoulos NG (2006) Propagating waves mediate information transfer in the motor cortex. Nat Neurosci 9:1549-1557.

Rudolph M, Destexhe A (2001) Correlation detection and resonance in neural systems with distributed noise sources. Phys Rev Lett 86:3662-3665.

Sakurai Y, Takahashi S (2006) Dynamic synchrony of firing in the monkey prefrontal cortex during working-memory tasks. J Neurosci 26:10141-10153.

Shadlen MN, Newsome WT (1998) The variable discharge of cortical neurons: implications for connectivity, computation, and information coding. J Neurosci 18:3870-3896.

Steinmetz PN, Roy A, Fitzgerald PJ, Hsiao SS, Johnson KO, Niebur E (2000) Attention modulates synchronized neuronal firing in primate somatosensory cortex. Nature 404:187-190.

Stark E, Abeles M (2007) Predicting movement from multiunit activity. J Neurosci 27:8387-8394.

Stark E, Asher I, Abeles M (2007) Encoding of reach and grasp by single neurons in premotor cortex is independent of recording site. J Neurophysiol 97:3351-3364.

Supèr H, Roelfsema PR (2005) Chronic multiunit recordings in behaving animals: advantages and limitations. Prog Brain Res 147:263-282.

Tetzlaff T, Rotter S, Stark E, Abeles M, Aertsen A, Diesmann M (2008) Dependence of neuronal correlations on filter characteristics and marginal spike-train statistics. Neural Comput 20:2133-2184.

Toyama K, Kimura M, Tanaka K (1981) Cross-correlation analysis of interneuronal connectivity in cat visual cortex. J Neurophysiol 46:191-201.

Treisman A (1996) The binding problem. Curr Opin Neurobiol 6:171-178.

Triesch J, von der Malsburg C (1996) Binding: a proposed experiment and a model. In: Proceedings of the international conference on artificial neural networks (von der Malsburg C, Seelen WV, Vorbruggen JC, Sendhoff B, eds). Berlin: Springer.

Ts'o DY, Gilbert CD, Wiesel TN (1986) Relationships between horizontal interactions and functional architecture in cat striate cortex as revealed by cross-correlation analysis. J Neurosci 6:1160-1170.

Vaadia E, Haalman I, Abeles M, Bergman H, Prut Y, Slovin H, Aertsen A (1995) Dynamics of neuronal interactions in monkey cortex in relation to behavioural events. Nature 373:515-518.

von der Malsburg C (1981) The correlation theory of brain function. MPI biophysical chemistry, Internal report 81-2. Reprinted in: Models of neural networks II, Chap 2 (1994) (Domany E, van Hemmen JL, Schulten K, eds), pp 95-119. Berlin: Springer.

Womelsdorf T, Schoffelen JM, Oostenveld R, Singer W, Desimone R, Engel AK, Fries P (2007) Modulation of neuronal interactions through neuronal synchronization. Science 316:1609-1612.

Zeitler M, Fries P, Gielen S (2006) Assessing neuronal coherence with single-unit, multi-unit, and local field potentials. Neural Comput $18: 2256-2281$

Zohary E, Shadlen MN, Newsome WT (1994) Correlated neuronal discharge rate and its implications for psychophysical performance. Nature 370:140-143. 